Rev. salud público. Sup. 8 (1): 59-70, 2006

\title{
Canales Endémicos y Marcadores de Resistencia Bacteriana, en Instituciones de Tercer Nivel de Bogota, Colombia
}

\author{
Aura Lucia Leal ${ }^{1}$, Javier Eslava Schmalbach ${ }^{2}$, Carlos Álvarez ${ }^{3}$, Giancarlo Buitrago ${ }^{4}$, \\ Matilde Méndez ${ }^{5}$ y Grebo* \\ ${ }^{1}$ Médico. M. Sc. Control de Enfermedades Infecciosas. Especialista en Microbiología y Parasitología \\ Médicas. Departamento de Microbiología, Facultad de Medicina. Universidad Nacional de Colombia. \\ E-mail: allealc@unal.edu.co \\ ${ }^{2}$ Médico. M. Sc. Epidemiología Clínica. Ph.D (Candidato). Instituto de Investigaciones Clínicas. Fa- \\ cultad de Medicina. Universidad Nacional de Colombia. E-mail: jheslavas@unal.edu.co \\ ${ }^{3}$ Médico. Especialista en Infectología. Unidad de Infectología, Hospital Universitario San Ignacio. \\ Facultad de Medicina, Universidad Nacional de Colombia. E-mail: caalvarezmo@unal.edu.co \\ ${ }^{4}$ Médico. Facultad de Medicina. Universidad Nacional de Colombia. E-mail: gcbuitrago@grebo.org \\ ${ }^{5}$ Bacterióloga. Hospital Militar Central. E-mail: mattymendez@cable.net.co \\ * Grupo para el Control de la Resistencia Bacteriana en Bogotá.
}

Recibido 28 Septiembre 2005/Enviado para Modificación 3 Marzo 2006/Aceptado 19 Abril 2006

\section{RESUMEN}

Objetivo Determinar los perfiles de resistencia bacteriana y los canales endémicos en 14 instituciones de tercer nivel.

Métodos Población. Bogotá-Colombia, 14 hospitales pertenecientes al Grupo para el Control de la Resistencia Bacteriana de Bogotá (GREBO). A partir de la información obtenida de los laboratorios de microbiología de los centros participantes (métodos automatizados y manuales), se creó una base de datos usando los programas BacLink 2.0 y Whonet 5.3, durante los años 2001, 2002 y 2003. Los perfiles de susceptibilidad fueron hallados acordes a las normas de la NCCLS (2003). Se realizó un análisis descriptivo de los diferentes marcadores de resistencia y se determinó el canal endémico de la resistencia para los hospitales, utilizando los puntos entre los percentiles 25 y $75 \%$, para cada mes durante el periodo de estudio.

Resultados Se analizaron 84664 aislamientos. Los más frecuentes fueron Escherichia coli, Staphylococcus aureus, Staphylococcus coagulasa negativo, Klebsiella pneumoniae y Pseudomonas aeruginosa. La resistencia para los años 2001, 2002 y 2003 fue respectivamente: S. aureus meticilino resistente: $41 \%, 48 \%, 48 \%$; Staphylococcus coagulasa negativo resistente a oxacilina: $75 \%, 73 \%, 72 \%$; E. faecium vancomicina resistente: $14 \%, 9 \%$, 
$3 \% ; K$. pneumoniae resistente a cefalosporinas de tercera generación: 37 $\%, 25 \%, 23 \%$; $P$. aeruginosa resistente a imipenem: $24 \%, 22 \%, 17 \%$; $P$. aeruginosa resistente a ciprofloxacina: $46 \%, 46 \%, 35 \%$, A. baumannii resistente a imipenem: $11 \%, 29 \%, 39 \%$. Los canales endémicos evidenciaron la problemática de la resistencia bacteriana, esta se centró en la pre sencia de $S$. aureus meticilino resistente y en el marcado incremento de la resistencia de $A$. baumanni a imipenem.

Conclusiones Se destacan los altos porcentajes de resistencia para todos los marcadores de impacto epidemiológico a nivel hospitalario especialmente en Unidades de Cuidado Intensivo.

Palabras Clave: Colombia, vigilancia epidemiológica (fuente: DeCS, BIREME).

\section{ABSTRACT \\ Endemic channels and bacterial resistance markers in third-level hospitals in Bogotá, Colombia}

Objective Determining antimicrobial resistance profiles and endemic channels in 14 third-level hospitals.

Methods A high complexity hospital network was created between 2001 and 2003 in Bogotá, Colombia, comprising 14 hospitals belonging to the Bogotá Bacterial Resistance Control Group (BBRCG) and a database was established from participating institutions' microbiology laboratory data (using automated and manual methods) using BacLink 2.0 and Whonet 5.3. Isolate susceptibility profiles were determined according to NCCLS (2003). A descriptive analysis was made of the different resistance markers and such resistance's endemic channel was determined for all hospitals using a $25 \%$ to $75 \%$ range for every month during the study period.

Results 84664 isolates were analysed, the most frequently found being Escherichia coli, Staphylococcus aureus, coagulase negative Staphylococcus, Klebsiella pneumoniae and Pseudomonas aeruginosa. S. aureus resistance to oxacillin in 2001, 2002 and 2003 was $41 \%, 48 \%$ and $48 \%$, respectively, Staphylococcus coagulasa negative resistance to oxacillin $75 \%, 73 \%$ and $72 \%$, E. faecium resistance to vancomycin was $14 \%, 9 \%, 3 \%$, K. pneumoniae resistance to third-generation cephalosporins $37 \%, 25 \%$ and $23 \%$, $P$. aeruginosa resistance to imipenem $24 \%, 22 \%$ and $17 \%, P$. aeruginosa resistance to ciprofloxacin $46 \%, 46 \%$ and $35 \%$ and $A$. baumannii resistance to imipenem $11 \%, 29 \%$ and $39 \%$, respectively. The problem of bacterial resistance became evident in the endemic channels; this was centred on the presence of oxacillin-resistant $S$. aureus and a marked increase in $A$. baumanni resistance to imipenem.

Conclusions High resistance levels were observed in epidemiologic impact markers, especially in Intensive Care Units. 
Key Words: Microbial drug resistance, Colombia, epidemiological surveillance (source: $\mathrm{MeSH}, \mathrm{NLM}$ ).

L

a emergencia y diseminación de la resistencia bacteriana, es considerada actualmente como un fenómeno creciente alrededor del mundo y de gran complejidad (1). Es por esto, que la Organización Mundial de la Salud (2), mediante resolución de 1998 la declaró como problema de Salud Pública y por tanto ha venido trabajando en la creación de una estrategia global, cuyos objetivos fundamentales, mediante la creación de una serie de intervenciones, son, estimular la prevención y control de infecciones, retardar la emergencia de resistencia y reducir la diseminación de microorganismos resistentes (3).

Dentro de las estrategias propuestas para contener la resistencia bacteriana, se plantean, el establecimiento y fortalecimiento de los programas de control de infección, la creación de programas de control en el uso de antimicrobianos y el establecimiento de normas regulatorias y de educación sobre el manejo de los mismos (4). Sin embargo, es la vigilancia de la resistencia bacteriana, la estrategia fundamental e inicial para el desarrollo de los procesos de control de este problema. La vigilancia de la resistencia, es por tanto esencial para proveer información sobre la magnitud y las tendencias de la resistencia y para monitorear el efecto de las medidas de intervención (5).

La información global sobre los perfiles de resistencia en Bogotá no se conocía. Existían datos aislados de los diferentes hospitales pero no se contaba con un sistema unificado para colección y análisis de la información que permita determinar la magnitud del problema y comparar la situación con otras ciudades del país. El grupo para el control de la resistencia bacteriana en Bogota (GREBO) está formado por hospitales de tercer nivel integrados en una red, con criterios unificados en la colección y análisis de información. Todos los hospitales disponen de un comité de infecciones y de un laboratorio que realice procedimientos estándares con control de calidad, que garanticen la validez de sus resultados.

El objetivo de este trabajo es, mediante la conformación de una red de vigilancia basada en el laboratorio, determinar los perfiles de resistencia generales y ver la progresión de esta mediante canales endémicos de los marcadores de resistencia bacteriana en los hospitales involucrados en la misma. 


\section{MÉTODOS}

Diseño

Estudio descriptivo sobre una cohorte de vigilancia epidemiológica, de todos los asilamientos bacterianos durante los años 2001, 2002 y 2003, en los hospitales participantes y que hacían parte del Grupo para el Control de la Resistencia Bacteriana de Bogotá (GREBO).

Los hospitales participantes consolidaron mensualmente la información de los datos de resistencia bacteriana de los pacientes de las unidades de cuidado intensivo (UCI) y de las otros servicios de hospitalización no UCI (NoUCI). El análisis bacteriológico se hizo utilizando métodos automatizados (MicroScan o Vitek), o manuales. Las bases de datos de cada institución fueron transferidas por el software BacLink $2.0 \mathrm{y}$ analizadas por el software Whonet (versión 5.3), de la Organización Mundial de la Salud. Se creó para ello un laboratorio global de la red GREBO.

Población

Microorganismos recuperados de pacientes atendidos en 14 instituciones de tercer nivel de Bogotá, involucradas en la red. Las instituciones participantes fueron: Clínica del Niño (183 camas), Clínica de Occidente (100 camas), Clínica San Pedro Claver (850 camas), Fundación Cardio Infantil (300 camas), Fundación San Carlos (100 camas), Hospital El Tunal (214 camas), Hospital Militar Central (650 camas), Hospital del Occidente Kennedy (200 camas), Hospital San José (183 camas), Hospital Simón Bolívar (360 camas), Hospital Universitario Clínica San Rafael (300 camas), Hospital Universitario San Ignacio (320 camas), Instituto Nacional de Cancerología (160 camas) y Hospital Santa Clara (200 camas).

Susceptibilidad bacteriana y control de calidad Microbiológica

La variable desenlace principal fue la resistencia bacteriana utilizando los puntos de corte del Comité Nacional de Estándares de Laboratorio de los Estados Unidos (NCCLS) para el año 2003 (6). Todos los laboratorios fueron sometidos a un programa de calidad externo, llevado a cabo por el laboratorio de Microbiología del Instituto Nacional de Salud.

Análisis

Para la información de resistencia bacteriana, se utilizó el programa Whonet (opción Uno por Paciente-sólo el primer aislamiento). Se realizó un análisis 
descriptivo de los diferentes marcadores de resistencia, y se determinó el canal endémico de la resistencia para los hospitales, utilizando los puntos entre los percentiles 25 y $75 \%$, para cada mes durante el periodo de estudio (7), y con él, se compararon cada una de las instituciones involucradas de manera anónima. Se utilizó el paquete estadístico STATA versión 8.1.

\section{RESULTADOS}

Durante los tres años del estudio, se recolectaron 84664 aislamientos provenientes de servicios UCI, NO UCI y extrahospitalarios. Los aislamientos de NO UCI, correspondieron a 65,9 \% del total de aislamientos ( $\mathrm{n}=55$ 809) y los de UCI, correspondieron a $21,7 \%$ del total ( $\mathrm{n}=18$ 407).

Figura 1. Distribución de los organismos aislados con mayor frecuencia en las Instituciones participantes Bogotá 2001-2003 n: 84664

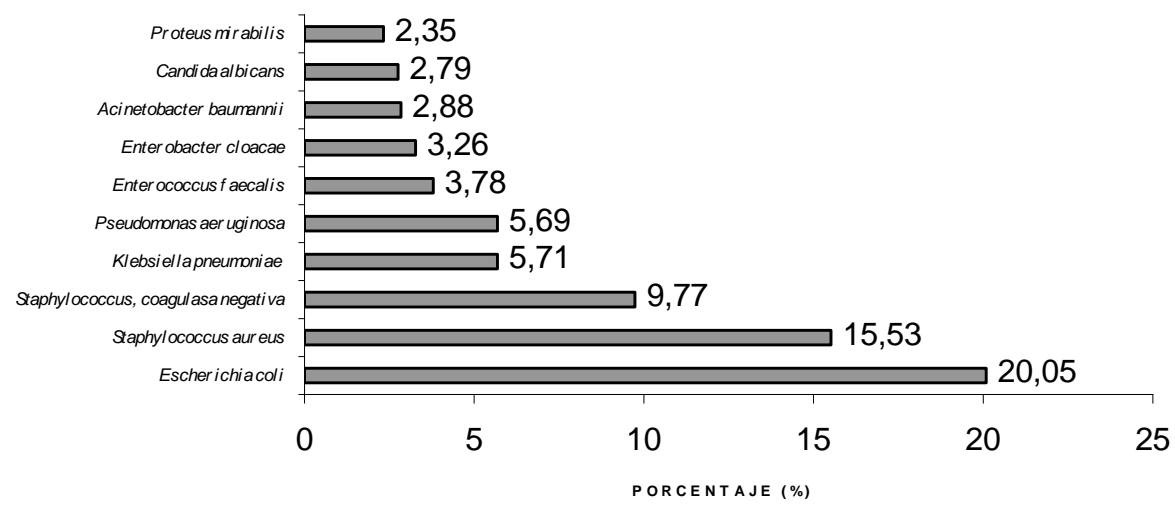

Los principales tipos de muestra de donde se obtuvieron los aislamientos fueron: orina (22,9\%), sangre (18,3\%), secreciones no especificadas $(17,8$ $\%)$, catéter (6,3\%), líquido abdominal (5,1\%), esputo (3,7\%) y heces (3,5 $\%)$.

Los siete microorganismos aislados con mayor frecuencia fueron Escherichia coli, Staphylococcus aureus, Staphylococcus epidermidis, Klebsiella pneumoniae, Pseudomonas aeruginosa, Enterococcus faecalis y Enterobacter cloacae (Figura 1). 
En la Tabla 1, se observan los porcentajes de resistencia de los marcadores de impacto epidemiológico durante los tres años. Se destacan los altos porcentajes de resistencia en general para todos los marcadores (con menor proporción para Enterococcus resistente a vancomicina). Este fenómeno fue especialmente notorio en Unidades de cuidado Intensivo. La resistencia de A. baumannii a imipenem pasó de $11 \%$ en el 2001 a $39 \%$ en el 2003. S. aureus y Staphylococcus coagulasa negativa resistente a oxacilina se caracterizó por presentar altos niveles de resistencia especialmente en UCI durante todo el periodo. Se presentaron elevados porcentajes de resistencia en $P$. aeruginosa frente a ciprofloxacina. E. cloacae frente a cefalosporinas de tercera generación. La resistencia de K. pneumoniae y E. coli a cefalosporinas de tercera generación como marcadores de potenciales productoras de beta lactamasas de espectro extendido (BLEE), aunque representó un fenómeno importante tuvo tendencia a disminuir en la mayoría de los hospitales de la ciudad.

Tabla 1. Marcadores de Resistencia de GREBO. Años 2001-2003

\begin{tabular}{lccc}
\hline \multicolumn{1}{c}{ Marcadores } & \multicolumn{2}{c}{ GREBO } \\
\cline { 2 - 3 } & 2001 & 2002 & 2003 \\
\hline A. baumannii, resistente a imipenem & 10,9 & 29,1 & 38,8 \\
\hline E. cloacae, resistente a cefalosporina de 3ra & 41 & 46,2 & 51,7 \\
\hline E. coli, potencial BLEE & 4,5 & 3,5 & 4,5 \\
\hline E. coli, resistente a ciprofloxacina & 19,8 & 21 & 20,3 \\
\hline E. faecium, resistente a vancomicina & 14,2 & 9,3 & 2,9 \\
\hline K. pneumoniae, potencial BLEE & 37,5 & 25,1 & 22,7 \\
\hline K. pneumoniae, resistente a ciprofloxacina & 12,2 & 8,2 & 7 \\
\hline P. aeruginosa, resistente a ceftazidima & 31,8 & 27,8 & 21,4 \\
\hline P. aeruginosa, resistente a ciprofloxacina & 46,4 & 45,7 & 34,9 \\
\hline P. aeruginosa, resistente a imipenem & 23,6 & 21,9 & 17,3 \\
\hline S. aureus, resistente a oxacilina & 41,1 & 48,2 & 48 \\
\hline S coagulasa negativo, resistente a oxacilina & 75,5 & 72,7 & 72,2 \\
\hline
\end{tabular}

Canales Endémicos

En la Figura 2 se muestra la tendencia en la resistencia de Staphylococcus aureus meticilino resistente (SAMR) y Enterococcus faecium vancomicino resistente (EVR). Durante el periodo de estudio fue evidente la presentación de brotes de EVR durante los meses junio noviembre del 2001, marzo mayo 2002, julio noviembre del 2002 y enero del 2003. La tendencia del SAMR 
fue la de aumentar la resistencia en el periodo de estudio sin que se puedan apreciar picos epidémicos.

Figura 2. Canal endémico de $S$. aureus resistente a oxacilina y tendencia de $E$. faecium resistente a vancomicina. GREBO. 2001-2003
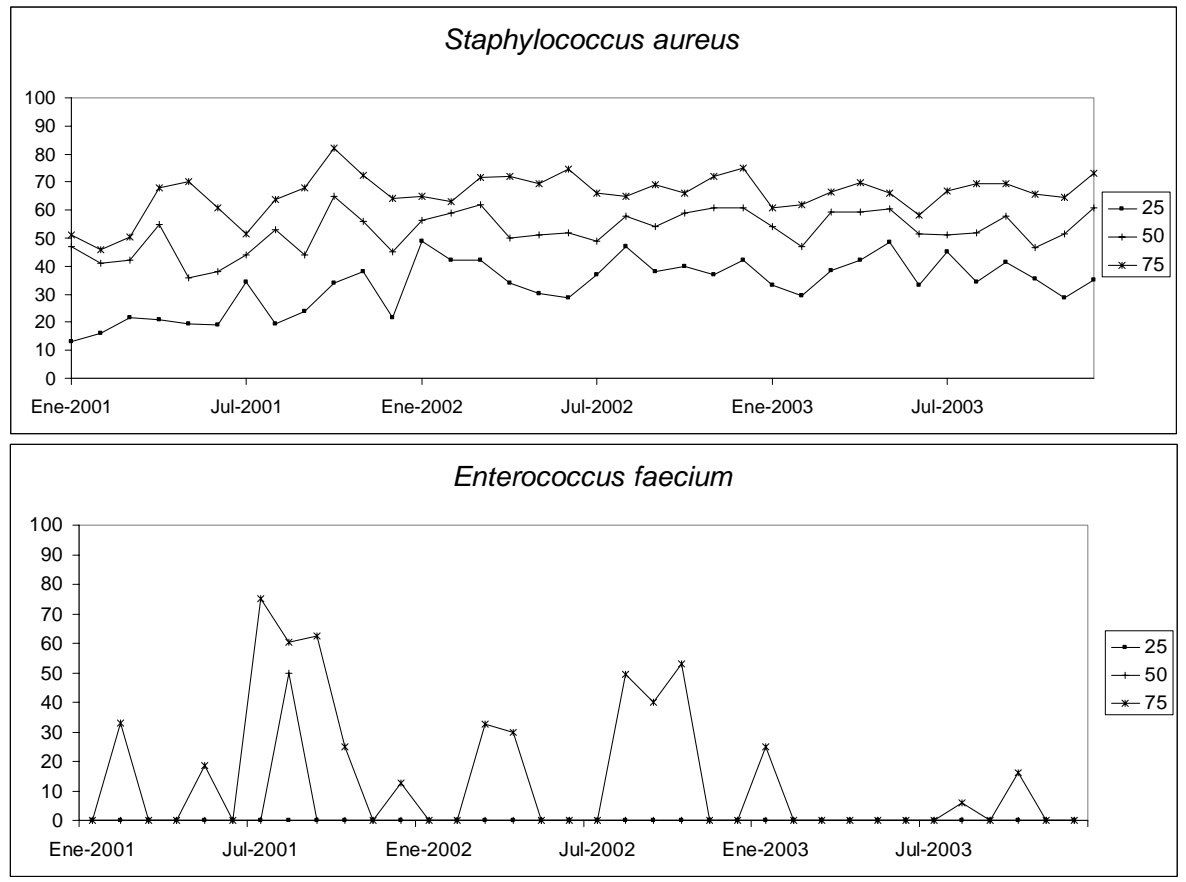

En la Figura 3 se aprecia el comportamiento en los perfiles de resistencia durante el período de estudio para E. coli y K. pneumoniae a ceftazidima. El comportamiento para la E. coli durante el periodo de estudio es estable, apreciándose un pico epidémico para los períodos Julio del 2002, noviembre del 2002, marzo y noviembre del 2003. Es llamativo un punto epidémico para $K$. pneumoniae resistente a ceftazidima en el período de septiembre del 2001, que repite en enero del 2002 y marzo mayo del 2003.

Con respecto a $E$. cloacae resistente a ceftazidima (canal endémico no mostrado) se aprecia un canal amplio con una gran dispersión de los datos y picos epidémicos para noviembre del 2002, enero del 2003 y marzo del 2003.

E. coli resistente a ciprofloxacina (canal endémico no mostrado), presenta oscilaciones en la resistencia que van entre un 10 y $44 \%$ (marzo del 2001), 16 a 45 \% (agosto del 2001), 20 a 45 \% (noviembre 2002), 20 a 37 \% (julio 
del 2003), que corresponden todos a momentos epidémicos dentro del canal endémico. Se observa una disminución del canal para diciembre del 2003 con valores entre 7 y $25 \%$ de resistencia

Figura 3. Canal endémico de $E$. coli y $K$. pneumoniae resistente a ceftazidima. GREBO. $2001-2003$
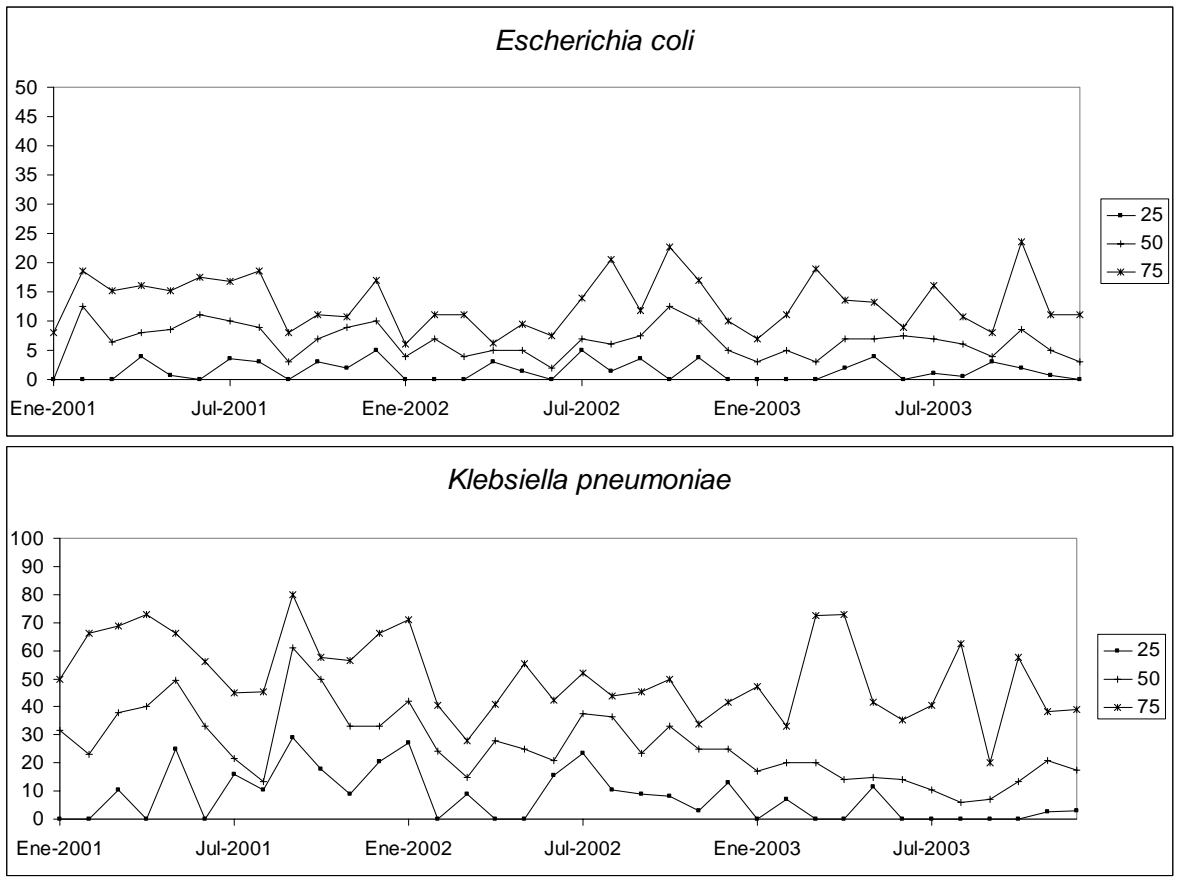

En la Figura 4 se aprecia el canal endémico de resistencia a imipenem de $P$. aeruginosa y A. baumannii. Para $P$. aeruginosa hay una tendencia global a la disminución de la resistencia durante el periodo de estudio con una discreta disminución en la amplitud del canal. En el caso del A. baumannii hay un aumento marcado en la resistencia global durante los tres años con un aumento en la amplitud del canal. A finales del año 2002 y durante el 2003 se aprecian picos epidémicos en la resistencia a Imipenem por parte de ambos gérmenes.

El canal endémico de la resistencia de $P$. aeruginosa a ceftazidima y ciprofloxacina (canal endémico no mostrado), muestra para ceftazidima una tendencia a mantener el patrón de resistencia durante el periodo de estudio, con un comportamiento epidémico durante los meses de mayo, agosto y octubre del 2001 y abril del 2003. En el caso de ciprofloxacina, P. aeruginosa muestra una tendencia global a la disminución de la resistencia durante 
los tres años, siendo más importante para el año 2003, presenta picos epidémicos para junio, julio del 2001 y septiembre octubre del mismo año. La amplitud del canal endémico se mantiene constante para el periodo en estudio.

Figura 4. Canal endémico de $P$. aeruginosa y $A$. baumannii resistentes a imipenem GREBO. 2001-2003
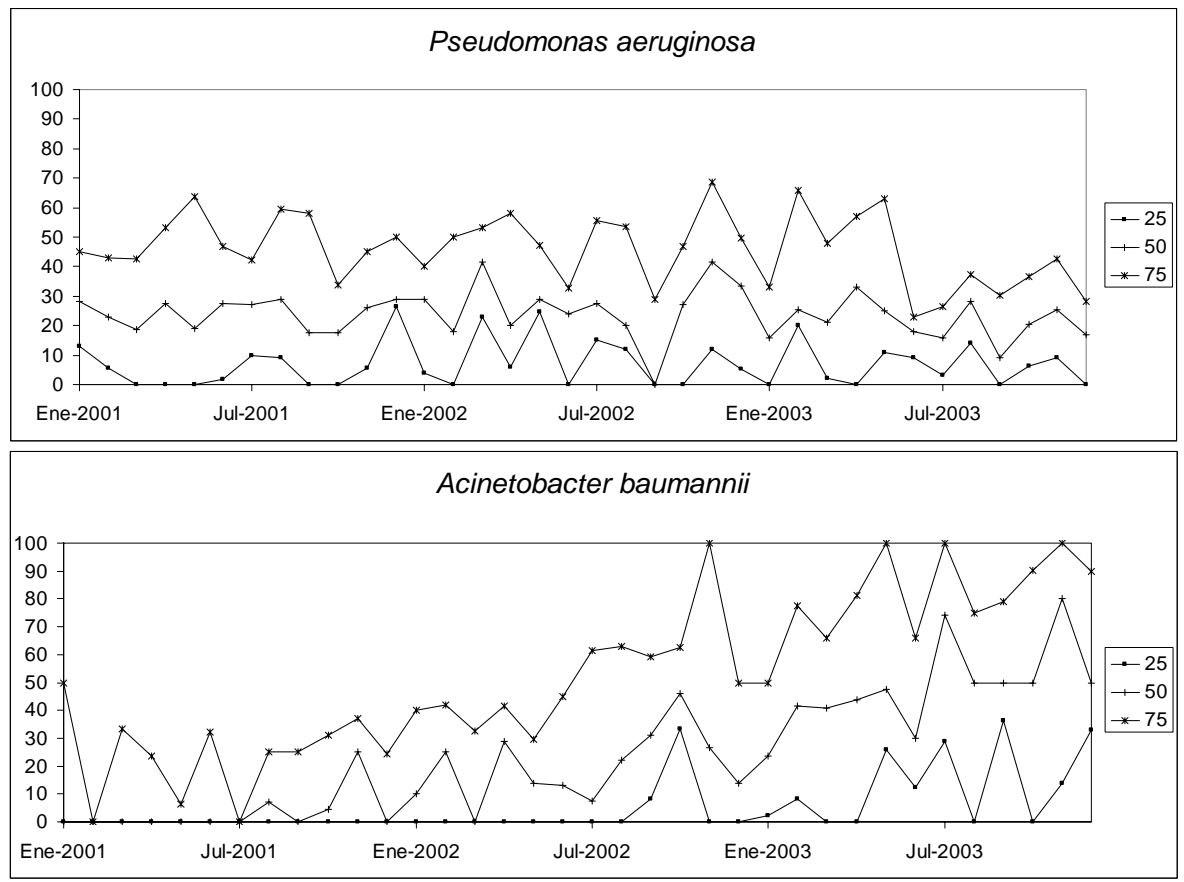

\section{DISCUSION}

La resistencia a los antimicrobianos es considerada un problema emergente para una variedad de microorganismos causantes de infecciones, tanto a nivel hospitalario como adquiridos en la comunidad. La presencia de multiresistencia ha llegado a ser común para organismos como Staphylococcus aureus, Enterococcus faecium, Streptococcus pneumoniae, especies de Acinetobacter (8), como se evidenció en este estudio.

En los países en desarrollo existen factores que contribuyen al incremento de la resistencia, entre ellos la ausencia de políticas claras para la formulación de antibióticos, la precaria situación de los sistemas de salud, el escaso 
número de investigaciones y la poca funcionalidad en muchos hospitales del sistema de vigilancia y control de las infecciones (3).

Dadas las características de los hospitales de esta red, y en particular de las unidades de cuidado intensivo, se pudo en evidencia que éstas últimas son una fuente continua e importante fuente de microorganismos resistentes. Esta sería la consecuencia de la exposición a altas cargas de antimicrobianos en una población en contacto frecuente con trabajadores de la salud y riesgo de infecciones cruzadas (9).

En los últimos años, en el mundo se han iniciado diferentes proyectos de vigilancia de la resistencia bacteriana como los proyectos SENTRY e ICARE $(10,11)$, los cuales han generado información valiosa con relación a la prevalencia de patrones de resistencia. En Colombia también se han iniciado esfuerzos por colectar información y determinar cual es la magnitud del fenómeno en el país. Es así como existe información de grupos de Bogotá, Valle y Antioquia. La información resultante de este proyecto, servirá de base para implementar políticas locales y regionales que permitan controlar la diseminación y facilitación de la resistencia, intra e interinstitucional, y disminuir el impacto que ella tiene sobre el uso de los recursos, cada vez más escasos, a nivel de las entidades gubernamentales, promotoras y prestadoras de salud.

El uso de de los canales endémicos ha sido muy útil como metodología para conocer la tendencia en el comportamiento de enfermedades parasitarias e infecciosas. Para ello se ha propuesto su construcción utilizando el promedio histórico del número de casos graficados en el tiempo, con su respectiva desviación estándar, para definir los bordes del canal. Para algunos de estos casos se eliminan los periodos que actuarían como datos extremos, tanto por picos o valles muy altos o bajos respectivamente $(12,13)$. También se ha propuesto el uso de la mediana con el rango intercuartílico, o el promedio con el uso de dos desviaciones estándar, el intervalo de confianza del 95 \% también se ha utilizado para este fin (14), y el uso de sistemas computarizados que calculan los canales con ajustes a través de modelos matemáticos complejos (15). Para este trabajo, y con la intención de eliminar el efecto que podrían tener sobre la tendencia de la endemicidad, los valores extremos epidémicos, se utilizo la mediana con el rango intercuartílico 25-75 \%.

La creación de redes como la que se presenta en este trabajo, es especialmente útil en países con recursos limitados y permite el análisis de las tendencias para poder detectar los cambios en los patrones de resistencia (16). El programan WHONET es una herramienta ideal para el análisis de 
los datos en forma conjunta y su compatibilidad con los sistemas automatizados permitió conocer la información local pero además la posibilidad de compartirla con otras redes a nivel nacional (17). La información generada servirá de base para proponer estrategias que permitan controlar los patrones de resistencia y por ende la infección en Bogotá.

Agradecimientos. Grupo para el Control de la Resistencia Bacteriana (GREBO) Hospitales e Instituciones participantes (2001-2003): Universidad Nacional de Colombia (Aura Lucía Leal Castro, Javier Eslava Schmalbach, Giancarlo Buitrago Gutiérrez, Carlos Saavedra Trujillo), Clínica del Niño (Mauricio Luna, Tailandia Rodríguez, Martha Uzeta), Clínica de Occidente (Elkin Lemos, Martha Salinas), Clínica San Pedro Claver (Carlos Alquichire, Martha Ruiz), Fundación Cardioinfantil (Álvaro Arango, Patricia Bravo), Fundación Universitaria San Carlos (Jorge A. Cortés, Jaime Saravia), Hospital El Tunal (Elkin Lemos, Narda Olarte, Martha Garzón), Hospital Militar Central (Matilde Méndez, Carlos Pérez), Hospital de Occidente Kennedy (Elkin Lemos, Romelia Villa), Hospital San José (Claudia Fajardo, Paola Jiménez), Hospital Santa Clara (Gloria Inés Gallo, Luzmila López, Roberto Támara), Hospital Simón Bolívar (Carlos Álvarez, Gustavo Aristizábal, Constanza Correa), Hospital Universitario San Ignacio (Carlos Álvarez, Diana Moncada), Hospital Universitario Clínica San Rafael (Clemencia Ávila, Carlos Saavedra, Martha Pulido), Instituto Nacional de Cancerología (Patricia Arroyo, Jorge A. Cortés y Sonia I. Cuervo), Asociación Colombiana de Infectología (ACIN) - Capítulo Central e Instituto Nacional de Salud.

\section{REFERENCIAS}

1. Tenover FC, Hughes JM. The challenges of emerging infectious diseases: development and spread of multiply-resistant bacterial pathogens. JAMA 1996;275:300-7.

2. World Health Organization. Containing Antimicrobial Resistant. Review of Literature and Report of Workshop on the developement of a Global Strategy for The Containment of antimicrobial Resistant. WHO/CDS /CRS/DRS/99.2 Genova; 1999.

3. World Health Organization's strategy to contain resistance to antimicrobial drugs. Rev Panam Salud Publica 2001; 10(4):284-94.

4. Shaes DM, Gerdng DN, Craig W, Bosnstein DL, Dunca RA, Eckman MR, et al. Society for healthcare epidemiology of America and Infectious diseases society of America joint committee on the prevention of antimicrobial resistance: guidelines for prevention of antimicrobial resistance in hospital. Clin Infect Dis J 1997; 25:584-99.

5. Mc Gowan JE: Antibiotic resistance in hospital bacteria: current patterns, modes of appearance or spread and economic impact. Rev Med Microbiol 1999; 2:161-9. 
6. National Committee for Laboratory Standards. Performance standards for susceptibility testing, 11 informational supplement. Approved standard M100-S10. Naitonal Committee for Clinical Laboratory standards, Wayne, Pa.; 2003.

7. Nájera JA, Koustnetsov RL, Delacollete C. Malaria Epidemics Detection and Control Forecasting and Pevention. WHO. [Internet] Disponible en: http://www.rbm.who.int/docs/najera_epidemics/naj10.htm. Consultado: Enero de 2005.

8. Tenover FC. Development and spread of bacterial resistance to antimicrobial agents: an overview. Clin Infect Dis 2001;33 Suppl 3:S108-15.

9. Flaherty JP, Weinstein RA. Nosocomial infection caused by antibiotic-resistant organisms in the intensive-care unit. Infect Control Hosp Epidemiol 1996;17(4):236-48.

10. Fridkin SK, Steward CD, Edwards JR, Pryor E, McGowan JE Jr, Archibald LK, Gaynes RP, Tenover FC. Surveillance of Antimicrobial Use and Antimicrobial Resistance in United States Hospitals: Project ICARE: Phase 2. Clin Infect Dis 1999; 29:245-52.

11. Diekema DJ, Pfaller MA, Schimitz FL, Smayevky J, Bell J, Jones NR, Beach M and the SENTRY participans group. Survey of infections due to Staphylococcus species: Frequency of occurrence and antimicrobial susceptibility of isolates collected in the Unites Sates, Canada, Latin America, Europe and the Pacific region for the SENTRY antimicrobial surveillance program 1997-1999. Clin Infect Dis 2001;32(suppl 2):S114-32.

12. Lloyd LS. Best Practices for dengue prevention and control in the Americas.OPS/OMS. US Agency for International Development. Washington, DC.; Febrero de 2003.

13. Fayad CV. Estadística Médica y de Salud Pública, Mérida, Venezuela, Universidad de los Andes, 1970. pp. 423-26.

14. Carrasco Asenjo M, Delgado García A, Fernández Pérez C, Prieto Valiente L, Jimeno Maestro J, Andradas Aragonés E. Epidemiologic surveillance of hospital infection. Preliminary analysis of a 5-year series. Med Clin (Barc) 1990;95(6):201-6.

15. Comas T, Diaz JC, Alonso AM. A computer system for epidemiological surveillance. Medinfo 1995;8 Pt 1:482.

16. Lewis D. Antimicrobial resistance surveillance: methods will depend on objetives. J Antimicrob Chemother 2002; 49:3-5.

17. O'Brien T, Eskildsen MA, Stelling JM. Using internet discussion of antimicrobial databases for continuos quality improvement of tha testing and management on antimicrobial resistance. Clin Infect Dis 2001;33 (Suppl 3):S118-23. 